\title{
Online meetings for the win
}

We welcome the move to virtual being the default for scientific interactions.

A $t$ the beginning of 2020, when the world was a different place, the editors of Nature Ecology \& Evolution started to think about setting firm targets for reducing our travel footprint. We had worked towards this informally over recent years - by avoiding some flights and giving more virtual presentations - but we had not set targets. Our initial planning included no flights for our UK-based editors travelling to our immediate European neighbours; making at least one of each editor's two or three annual lab visits a virtual one; and offsetting any necessary air travel. Needless to say, these discussions were rapidly overtaken by widespread travel bans, and we have ended up surpassing even the most stringent targets we could have set. No editors have travelled for work since February, and all of us have worked exclusively from home since early March.

In that time, the scientific community has moved an enormous proportion of its activity online. The early days of the pandemic saw the cancellation of most academic conferences, followed rapidly by the transfer of many conferences to an online format. Much of this adjustment built on the work of a small number of pioneering conferences that were already running online (see here for example). Seminar series have also moved online, not to mention entire teaching courses (a colossal effort that merits a separate discussion).

Although this came about through necessity, we welcome the coming of age of the virtual conference, and not just for the direct environmental impact. The traditional academic conference, without substantial change to format, location and financing, disadvantages many: those with more modest grant funding, researchers from the Global South, members of groups who are not safe or welcome in particular locations, scientists with disabilities, and individuals - predominantly women with childcare responsibilities. Virtual conferences are not a silver bullet to solve all these disparities, but they can substantially reduce many of the barriers.

We now need to decide how we want the landscape of academic conferences to look in the post-pandemic future. Many researchers have realized that even if conferences were to go back to the way they were (which they should not), they would drastically reduce the number they attend, either in person or online. But conferences are still of critical importance, especially to facilitate networking by early-career researchers and those from smaller institutions. We need to capitalize on the improvements that have been made to virtual events and keep the majority in that format, but would still expect that some will occur physically. Large conferences that run annually could be moved to a two- or three-year frequency, and/or a 'regional hub' approach could be adopted where the majority of people travel only short distances to attend an event linked to other hubs or to virtual attendees on different continents. At the very least, more scientific societies could adopt the established model of alternating global meetings with smaller regional ones. The ABCD framework (All continents, Balanced gender, low Carbon transport, Diverse backgrounds) for a mixed format offers further ideas for meeting schedules and structures.

We tip our hats to those who have already conducted online conferences, either as a pioneer before the pandemic, or more recently under immense uncertainty and time constraints. But how can virtual and mixed format conferences improve further? In recent months, the editors of Nature Ecology \& Evolution have attended several, spanning a range of disciplines, and we offer some anecdotal suggestions. One feature that has jumped out is the difficulty of attending a virtual conference outside of one's physical time zone. To some extent, scheduling inconvenience may be unavoidable but it can be mitigated by making recorded talks available after the conference (see also here), repeating sessions, and hosting virtual networking or Q\&A sessions at a range of different times to facilitate participants in all locations. One standout example is the International Institute for Sustainability Australia and the International Convention on Biological Diversity's ongoing webinar series which broadcasts identical live content at two different times, and simultaneously translated from English into French and Spanish.

Another casualty of virtual conferencing is in-person networking or introductions, which we editors along with everyone else find helpful for keeping up with our communities. Dedicated workshops or webinars on targeted discussion points can help address this. We particularly appreciated the European Society for the study of Human Evolution's use of the virtual interaction platform gather.town. Many participants spent the sessions accidentally barging in on the conversations of others, but this certainly worked as an ice-breaker, and is the closest we've seen to replicating the serendipitous nature of in-person chats during breaks.

Although online talks and approaching speakers in chat rooms might replace some of what editors do at conventional conferences, virtual conferences make it more challenging for researchers to approach us to discuss their projects or ask about the journal, things we try to encourage. Like the rest of the community, we are still working out how to make ourselves visible and approachable in virtual meetings, but please get in touch at any time via twitter, e-mail or within the conference platform - we love a (virtual) chat!

Seminars are also an important way of keeping the scientific community talking outside of annual meetings, and they have been relatively easy to move online. It now seems extraordinary how few were online and how many were in person just a year ago. Given the wider reach of online seminars, we suggest that these should remain the norm. Where an actual physical seminar occurs, with a visiting speaker, this should be as part of extensive collaborative discussion between the speaker and many local researchers, preferably over the course of several days to maximize benefit, and should definitely not be just a fly-in fly-out interaction.

As we start to look cautiously beyond the pandemic, we are committed to making our own interactions with the research community more sustainable, and support wider moves within our own industry. For example, Springer Nature, the publisher of Nature Ecology \& Evolution, has made a commitment to carbon neutrality for its business operations and employee flights. It is clear that scientific interactions are undergoing a long-overdue step change, and all stakeholders should embrace the virtual future.

Published online: 25 November 2020 https://doi.org/10.1038/s41559-020-01362-3 